\title{
Biomarcadores histológicos em Sciades herzbergii (Pisces, Ariidae) para avaliação de impactos em ambientes estuarinos da Baía de São Marcos, Maranhão
}

\author{
[Biomarkers histological in Sciades herzbergii (Pisces, Ariidae) for impact assessment in \\ estuarine environments of São Marcos Bay, Maranhão] \\ S.H.C. Soares ${ }^{1}$, D.B.P. Sousa ${ }^{2}$, W.B. Jesus ${ }^{1}$, R.N.F. Carvalho-Neta ${ }^{3}$ \\ ${ }^{1}$ Programa de pós-graduação - Universidade Estadual do Maranhão - São Luís, MA \\ ${ }^{2}$ Programa de pós-graduação - Rede Bionorte - Universidade Estadual do Maranhão - São Luís, MA \\ ${ }^{3}$ Universidade Estadual do Maranhão - São Luís, MA
}

\begin{abstract}
RESUMO
Neste estudo, objetivou-se avaliar o impacto ambiental em áreas estuarinas da Baía de São Marcos, Maranhão, utilizando-se alterações histológicas nas brânquias e no fígado de Sciades herzbergii como biomarcadores de contaminação aquática. Foram realizadas coletas no período chuvoso (março de 2018) e de estiagem (julho de 2018), em que foram capturados 40 exemplares de $S$. herzbergii em duas regiões distintas da Baía de São Marcos: A1) Ilha dos Caranguejos (área referência); A2) Terminal de Pesca Industrial de Porto Grande (área potencialmente contaminada). As estações foram georreferenciadas por GPS e, em cada local de captura dos peixes, registraram-se as seguintes variáveis abióticas: temperatura, salinidade, $\mathrm{pH}$ e oxigênio dissolvido. Em campo, os organismos foram pesados e medidos, as brânquias e os fígados foram retirados e fixados. Posteriormente, foram mantidos em álcool a $70 \%$ até o procedimento histológico padrão. Resultados indicam que as duas áreas são semelhantes em termos de características físico-químicas da água e do sedimento. Todavia, a análise semiquantitativa das alterações histológicas observadas revelou que as brânquias foram o órgão mais afetado nos peixes de $\mathrm{A} 2$, indicando que os peixes desse local apresentam danos teciduais maiores resultantes de estresse acentuado por impactos antrópicos.
\end{abstract}

Palavras-chave: alterações histológicas, brânquias, estuário, fígado, peixe

\begin{abstract}
This study aimed to evaluate the environmental impact in estuarine areas of São Marcos Bay, Maranhão, using histological changes in the gills and liver of Sciades herzbergii as biomarkers of aquatic contamination. Samples were collected during the rainy (March 2018) and dry (July 2018) seasons, in which 40 specimens of S. herzbergii were captured in two distinct regions of São Marcos Bay: A1) Crab Island (reference area); A2) Porto Grande Industrial Fishing Terminal (potentially contaminated area). The stations were georeferenced by GPS and at each fish capture site the following abiotic variables were recorded: temperature, salinity, $p H$ and dissolved oxygen. In the field, the organisms were weighed and measured and the gills and livers removed and fixed. Afterwards, they were kept in 70\% alcohol until the standard histological procedure. Results indicate that the two areas are similar in terms of physicochemical characteristics of water and sediment. However, the semiquantitative analysis of the observed histological alterations revealed that the gills were the most affected organ in A2 fish, indicating that the fish in this site have greater tissue damage resulting from stress accentuated by anthropic impacts.
\end{abstract}

Keywords: histological changes, gills, estuary, liver, fish

\section{INTRODUÇÃO}

Os estuários são ecossistemas de elevada importância ecológica e socioeconômica, uma vez que são utilizados como vias de acesso para o interior do continente (Pereira Filho et al., 2003). No entanto, devido a sua proximidade com a costa, esses ambientes estão submetidos a riscos de poluição em um ritmo preocupante decorrente da emissão de vários estressores antropogênicos

Recebido em 9 de setembro de 2019 
liberados por fontes industriais e domésticas (Stentiford et al., 2003). Portanto, é necessário o monitoramento contínuo para avaliar a situação de contaminação nessas regiões costeiras, em especial a saúde dos peixes que integram todo o histórico de poluição do ambiente aquático (Pinheiro Sousa et al., 2019).

Nesse contexto, o biomonitoramento torna-se um dos principais meios para investigar os processos resultantes das interações entre as misturas de substâncias tóxicas e a biota, que atuam sinergicamente e antagonicamente no compartimento hídrico (Prabhakaran et al., 2017). Para tanto, essa abordagem científica utiliza os biomonitores, compreendidos como os organismos aquáticos amostrados cuja análise de suas respostas biológicas ou biomarcadores indica graus de mudanças nos diferentes níveis da organização biológica (Rainbow, 1995). Os biomarcadores histológicos têm sido propostos como uma excelente metodologia de monitoramento para avaliar a sanidade dos organismos aquáticos, visto que as alterações histológicas aparecem, em médio prazo, a estressores subletais; essa abordagem proporciona um método rápido para detectar efeitos irritantes em vários tecidos e órgãos (Bernet et al., 1999), especialmente em espécies aquáticas nativas de ambientes tropicais (Fortes Carvalho Neta et al., 2019; Pinheiro Sousa et al., 2019).

No Brasil, vários estudos demonstraram a utilidade de metodologias que utilizam biomarcadores histológicos, com o intuito de avaliar efeitos biológicos de contaminantes em espécies capturadas em águas costeiras (Sousa et al., 2013; Sardi et al., 2016). Nas regiões Norte e Nordeste, pesquisas dessa abrangência são crescentes, principalmente em áreas próximas a instalações portuárias (Carvalho-Neta et al., 2012; Castro et al., 2018; Castro et al., 2019). Além disso, frequentemente, tem-se observado que os peixes são selecionados como importantes modelos biológicos, com a finalidade de investigar as respostas biológicas desses organismos resultantes das interações com os xenobióticos.

No Maranhão, a espécie de bagre estuarino Sciades herzbergii é recurso pesqueiro abundante e de relevância comercial na pesca artesanal do estado, sendo, normalmente, capturado em uma extensa Baía, localizada no Golfão Maranhense, denominada de Baía de São Marcos, onde estão inseridas áreas protegidas por lei, mas também portos de alta complexidade, historicamente impactados por metais pesados e hidrocarbonetos policíclicos aromáticos (Carvalho-Neta et al., 2012; Fortes Carvalho-Neta et al., 2016; Fortes Carvalho-Neta et al., 2019). Esses estudos devem ser contínuos e envolver outras áreas de amostragem dos peixes, a fim de verificar a extensão dos impactos causados pelos empreendimentos portuários da região e, assim, subsidiar ações de gestão ambiental. Desse modo, no presente trabalho, objetivou-se avaliar o impacto ambiental em áreas da Baía de São Marcos, utilizando-se alterações histológicas nas brânquias e no fígado de Sciades herzbergii como biomarcadores de contaminação aquática.

\section{MATERIAL E MÉTODOS}

O protocolo de coleta em campo e de procedimentos laboratoriais deste estudo foi aprovado pelo Comitê de Ética institucional da Universidade Estadual do Maranhão (01/2018) e cumpriu com as diretrizes do Colégio Brasileiro de Experimentação Animal/ Sociedade Brasileira da Ciência em Animais de Laboratório - SBCAL/Cobea (SBCAL/Cobea, http://www.sbcal.org.br). A licença ambiental foi obtida junto ao órgão gestor da Unidade de Conservação, Secretaria Estadual de Meio Ambiente e Recursos Naturais - Sema (015/2019).

Foram capturados 40 espécimes de Sciades herzbergii em duas áreas distintas da Baía de São Marcos, Maranhão (Fig. 1), no período de março e julho de 2018. A Ilha dos Caranguejos (A1 = $2^{\circ} 50$ '261" S/44³0'614" O) foi utilizada como área de referência por ser um local desabitado que não apresenta instalações urbanas e industriais e estar inserida em uma Unidade de Conservação de Uso Sustentável, denominada de Área de Proteção Ambiental da Baixada Maranhense (APA).

A região de influência do Complexo Portuário de São Luís, Maranhão, conhecida como Terminal de Pesca Industrial de Porto Grande (A2 = $2^{\circ} 45^{\prime} 775^{\prime \prime} \mathrm{S} / 44^{\circ} 21^{\prime} 285^{\prime \prime} \mathrm{O}$ ), foi considerada uma área potencialmente contaminada, por estar localizada próximo ao Porto do Itaqui, com um grande fluxo de embarcações e desembarques que atendem às cargas gerais e aos derivados de petróleo do estado. 


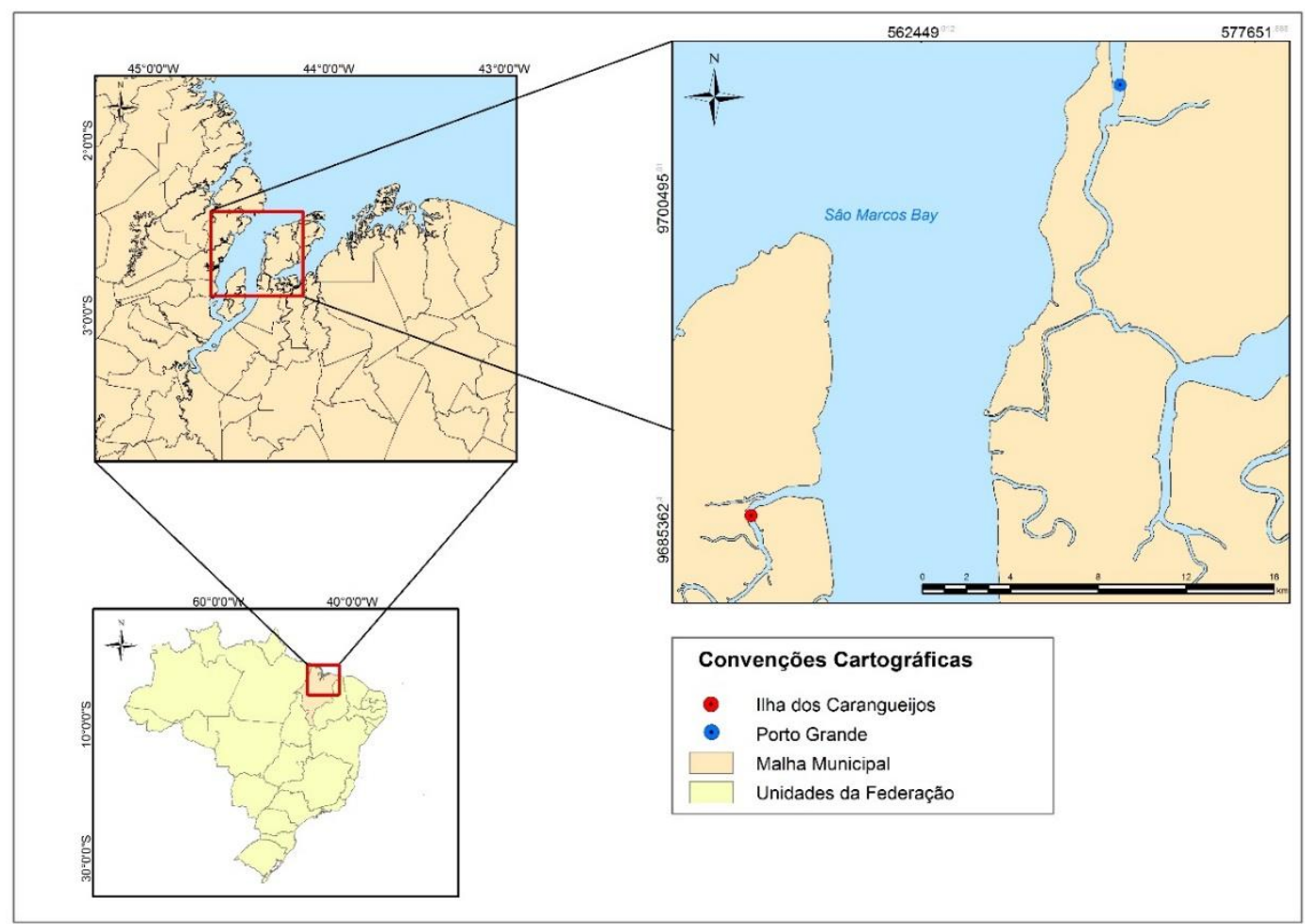

Figura 1. Região de coleta dos espécimes de Sciades herzbergii na Baía de São Marcos, Maranhão, Brasil. Ilha dos Caranguejos (A1 - área de referência). Terminal de Pesca Industrial de Porto Grande (A2 - área potencialmente contaminada).

Nos locais de coleta, foram aferidos os dados de salinidade, temperatura, $\mathrm{pH}$ e oxigênio dissolvido, com auxílio de equipamento multiparâmetro (SX751 - SANXIN). Dos exemplares de $S$. herzbergii amostrados foram registrados os dados morfométricos de comprimento total (CT), comprimento padrão $(\mathrm{CP})$, comprimento furcal (CF), em centímetro, e peso total (PT), em grama. Depois de medidos e pesados, os peixes foram eutanasiados por secção da medula espinhal, conforme anexo 1 da Resolução do Conselho Federal de Medicina Veterinária No 1000/2012 (Conselho..., 2012). Posteriormente, os exemplares foram abertos por meio de incisão vertical, para observação e classificação macroscópica das gônadas. A identificação de machos e fêmeas foi feita de acordo com BrownPeterson et al. (2011).

Logo depois, foram retiradas as brânquias e o fígado de cada peixe, os quais foram fixados em solução de formalina a $10 \%$, durante 24 horas, e mantidas em álcool 70\%. O segundo arco branquial direito foi descalcificado em ácido nítrico a $10 \%$, por seis horas. Em seguida, foi realizado o processamento histológico padrão, que consistiu na desidratação crescente em álcoois, diafanização em xilol, impregnação e inclusão em parafina (Caputo et al., 2010). Para o procedimento histológico, cortes transversais de aproximadamente $5 \mu \mathrm{m}$ de espessura foram corados com hematoxilina e eosina (HE). A leitura das lâminas foi realizada em microscópio óptico utilizando as objetivas de 10x, 20x e 40x, e as lesões identificadas foram fotomicrografadas em fotomicroscópio Axioskop - Zeis.

As alterações histológicas branquiais e hepáticas foram avaliadas de forma semiquantitativa, de acordo com o sistema de pontuação de Bernet $e t$ al. (1999). Cada alteração histológica foi classificada em um dos quatro padrões de reação: distúrbios circulatórios $\left(\mathrm{Rp}_{1}\right)$, que correspondem a condições patológicas no fluxo sanguíneo e tecidual; mudanças regressivas $\left(\mathrm{Rp}_{2}\right)$, que são processos que causam diminuição funcional ou perda de um órgão; mudanças progressivas $\left(R p_{3}\right)$, que correspondem a processos que levam ao aumento da atividade celular ou tecidual; e resposta inflamatória $\left(\mathrm{Rp}_{4}\right)$, que são processos 
inflamatórios associados a outros padrões de reação. No final, foram gerados índices para cada padrão de reação de um órgão (IH org. Rp), os quais foram utilizados para fornecer um índice geral do órgão (IH brânquias = índice histológico das brânquias; IH fígado = índice histológico de fígado). Para determinar o estado geral da sanidade dos peixes com base nas lesões histológicas, um índice histológico total (IH total) foi calculado pelo somatório de índices branquiais e hepáticos de cada organismo. Logo, quanto maiores os valores dos índices, mais severamente os órgãos foram afetados.

Os dados obtidos (parâmetros abióticos, morfometria, histológicos) foram submetidos ao teste de normalidade por meio do teste de Kolmogorov-Smirnov $(\mathrm{P}<0,05)$. Posteriormente, foi aplicado o teste $t$ de Student, com o uso do software Statistica 7.0 (Statsoft Inc., 2005, Tulsa, OK, EUA), para determinar diferenças significativas entre os grupos (área de referência e área portuária), e apenas $\mathrm{P}<0,05$ foi considerado significante.

\section{RESULTADOS E DISCUSSÃO}

Os índices histológicos das brânquias variaram entre as áreas de coleta (Tab. 1). Os valores de IH branquiais. Rp2 foram maiores na região portuária, da mesma forma que IH branquiais. Rp3. Essas médias apresentaram diferenças significativas entre os locais de amostragem, com $\mathrm{P}=0,015$ e $\mathrm{P}=0,019$, respectivamente. Porém, tais diferenças não foram observadas entre os períodos sazonais. Em relação ao padrão de reação, que inclui as respostas inflamatórias $\left(R_{p 4}\right)$, não foram encontradas alterações branquiais.

As alterações histológicas dos padrões regressivos e progressivos contribuíram para os maiores valores da média do IH branquiais, ou índice do órgão, da região portuária $(\mathrm{P}=0,04)$, evidenciando, portanto, que os peixes desse local experimentam danos maiores decorrentes de um elevado estresse. Resultados similares foram encontrados por Rautenberg et al. (2015), ao estudarem biomarcadores histológicos em Gambusia affinis coletados em diferentes pontos na bacia do rio Suquía, Argentina. Os autores verificaram que os danos histológicos nessa espécie tiveram relação com os efeitos combinados de vários contaminantes na água do rio, em especial, os metais pesados, e que as brânquias dos indivíduos capturados nos locais de amostragem, inclusive a área considerada como referência, apresentaram alterações histológicas.

Entre os índices histológicos hepáticos calculados para os peixes capturados na Baía de São Marcos, somente o IH fígado. Rp1 foi significativamente diferente entre os períodos de coleta, apresentando os maiores valores na estação chuvosa $(\mathrm{P}=0,02)$. Segundo Qualhato et al. (2018), as alterações circulatórias são respostas das células sanguíneas a agentes tóxicos e normalmente estão associadas às respostas inflamatórias. Esses autores detectaram diversas lesões do padrão $R_{\mathrm{p} 1}$ e $R_{\mathrm{p} 4}$ no fígado da espécie Poecilia reticulata submetida a teste de toxicidade aguda, em que foi exposta a nanopartículas de óxido de ferro.

Lesões hepáticas características do padrão de reação de mudanças progressivas $\left(\mathrm{R}_{\mathrm{p} 3}\right)$ não foram detectadas. As maiores médias dos padrões de reação de alterações hepáticas compreenderam as do tipo mudanças regressivas, sugerindo que essas lesões correspondem a processos que causam a perda funcional do órgão. No entanto, não houve diferença significativa entre essas alterações para ambas as áreas de coleta. $\mathrm{O}$ mesmo fato ocorreu para o padrão $R_{p 4}$. Assim, esses resultados influenciaram no valor do índice do órgão (IH fígado), em que não houve diferença estatística entre as médias das regiões de estudo. Por último, o índice histológico total (IH total) mostrou valores significativamente maiores na região portuária, apresentando um padrão de variação semelhante ao IH brânquias, o que confirma que o os peixes amostrados nessa região sofrem um tipo de estresse mais grave (Tab.1).

Valores expressos como média \pm desvio-padrão. A1 (área referência); A2 (área potencialmente impactada); IH: índice histológico; Rp1: padrão de reação 1 (distúrbios circulatórios); Rp2: padrão de reação 2 (mudanças regressivas); Rp3: padrão de reação 3 (mudanças progressivas); Rp4: padrão de reação 4 (respostas inflamatórias); NE: não encontrado. Letras diferentes indicam diferenças significativas $(\mathrm{P}<0,05)$ entre os locais de amostragem ("a"/"e") e os períodos sazonais ("c"/"d"). 
Biomarcadores histológicos...

Tabela 1. Índices histológicos branquiais e hepáticos de Sciades herzbergii coletados em regiões estuarinas (A1 e A2) da Baía de São Marcos, Maranhão

\begin{tabular}{|c|c|c|c|c|}
\hline \multirow[t]{3}{*}{ Índices histológicos } & \multicolumn{4}{|c|}{ Locais de amostragem } \\
\hline & \multicolumn{2}{|c|}{ A1 } & \multicolumn{2}{|c|}{$\mathrm{A} 2$} \\
\hline & Chuvoso & Estiagem & Chuvoso & Estiagem \\
\hline IH brânquias/ Rp.1 & $2,33 \pm 0,81$ & $2,28 \pm 0,75$ & $2,36 \pm 0,8$ & $2,8 \pm 1,09$ \\
\hline IH brânquias/ Rp.2 & $4,33 \pm 3,44^{\mathrm{a}}$ & $6 \pm 3,16^{\mathrm{a}}$ & $8,8 \pm 2,28^{b}$ & $8,8 \pm 3,03^{b}$ \\
\hline IH brânquias/ Rp.3 & $5,6 \pm 4,56^{a}$ & $8 \pm 3,26^{\mathrm{a}}$ & $10,4 \pm 3,57^{b}$ & $9,6 \pm 3,57^{b}$ \\
\hline IH brânquias/ Rp.4 & $\mathrm{NE}$ & $\mathrm{NE}$ & $\mathrm{NE}$ & $\mathrm{NE}$ \\
\hline IH fígado/ RP.1 & $2 \pm 0^{c}$ & $2 \pm 0$ & $2,36 \pm 0,8^{d}$ & $2 \pm 0$ \\
\hline IH fígado/ Rp.2 & $2,5 \pm 1$ & $3 \pm 1,15$ & $3,16 \pm 0,98$ & $4 \pm 0$ \\
\hline IH fígado/ Rp.3 & $\mathrm{NE}$ & $\mathrm{NE}$ & $\mathrm{NE}$ & $\mathrm{NE}$ \\
\hline IH fígado/ Rp.4 & $2,85 \pm 1,06$ & $3 \pm 1,41$ & $2,57 \pm 0,97$ & $2,85 \pm 1,06$ \\
\hline IH brânquias & $22,6 \pm 7,57^{a}$ & $26 \pm 8,71^{a}$ & $40,6 \pm 13,31^{b}$ & $35,3 \pm 18,58^{b}$ \\
\hline IH fígado & $18 \pm 7,21$ & $11,33 \pm 5,03$ & $21 \pm 4,35$ & $17,33 \pm 2,3$ \\
\hline IH total & $24,4 \pm 8,76^{\mathrm{a}}$ & $22,4 \pm 10,71^{a}$ & $37 \pm 3,16^{b}$ & $31,6 \pm 9,42^{b}$ \\
\hline
\end{tabular}

Apesar de não se poder relacionar as alterações histológicas encontradas nesta pesquisa à presença de contaminantes específicos, verificase que as respostas biológicas da espécie $S$. herzbergii refletem diferentes níveis de estresse em regiões distintas da Baía de São Marcos (Fig. 2 e 3). As alterações identificadas diferenciaram claramente os dois ambientes analisados, tendo os peixes da região portuária apresentado um padrão de sanidade comprometido. A Ilha dos Caranguejos permanece sendo uma área de referência mediante a justificativa de não apresentar instalações urbanas e industriais nas suas proximidades. Entretanto, os resultados obtidos nesta pesquisa apontam que esse local não se encontra isento de impactos, que podem ter influência antrópica, porém em menor escala.
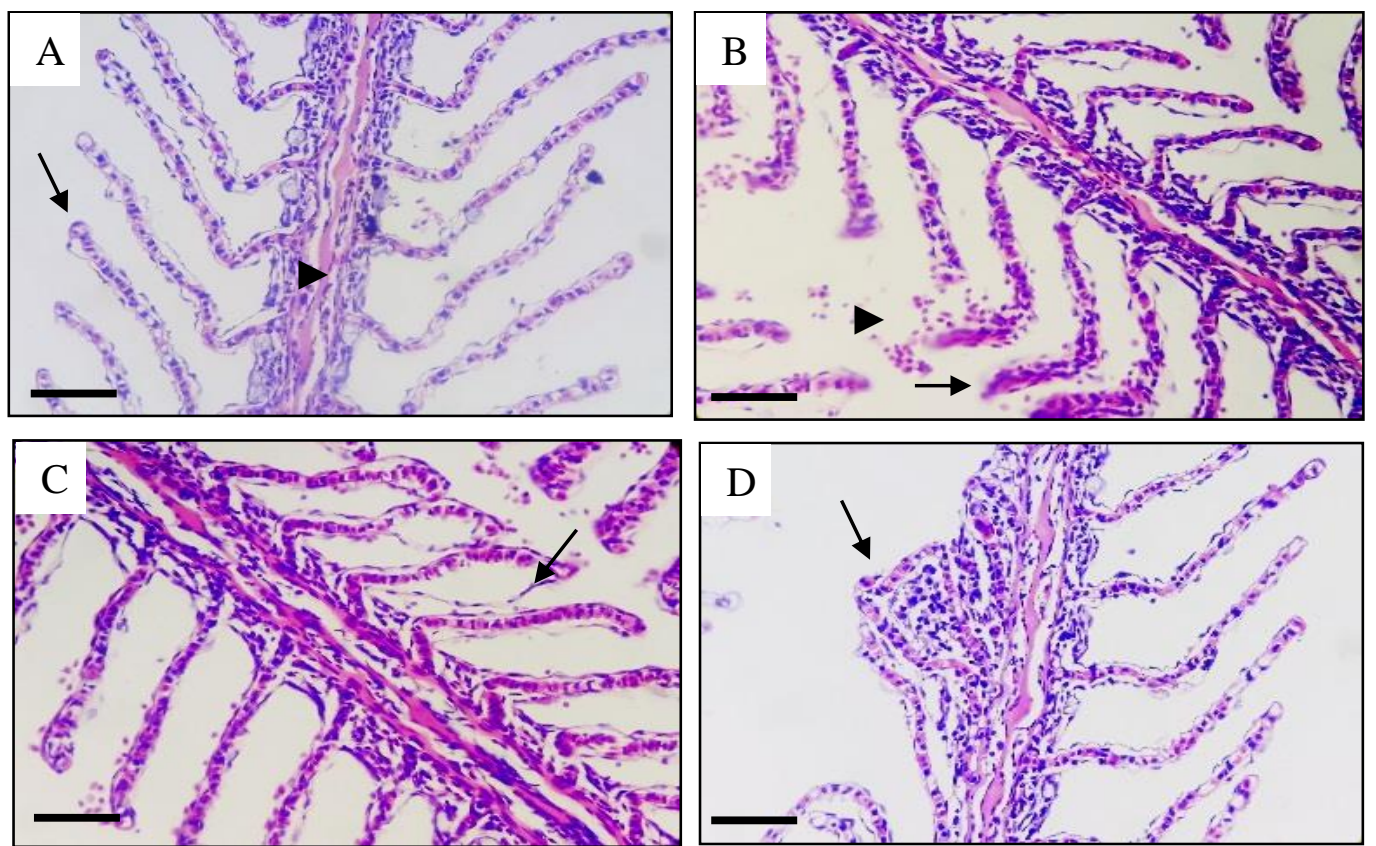

Figura 2. Fotomicrografias do segundo arco branquial direito de S. herzbergii. A) Filamento branquial (ponta de seta) e lamelas secundárias (seta) normais; B) hemorragia (ponta de seta) e congestão vascular (seta); C) levantamento epitelial (seta); D) fusão lamelar. Escala= 50 $\mu \mathrm{m}$. 

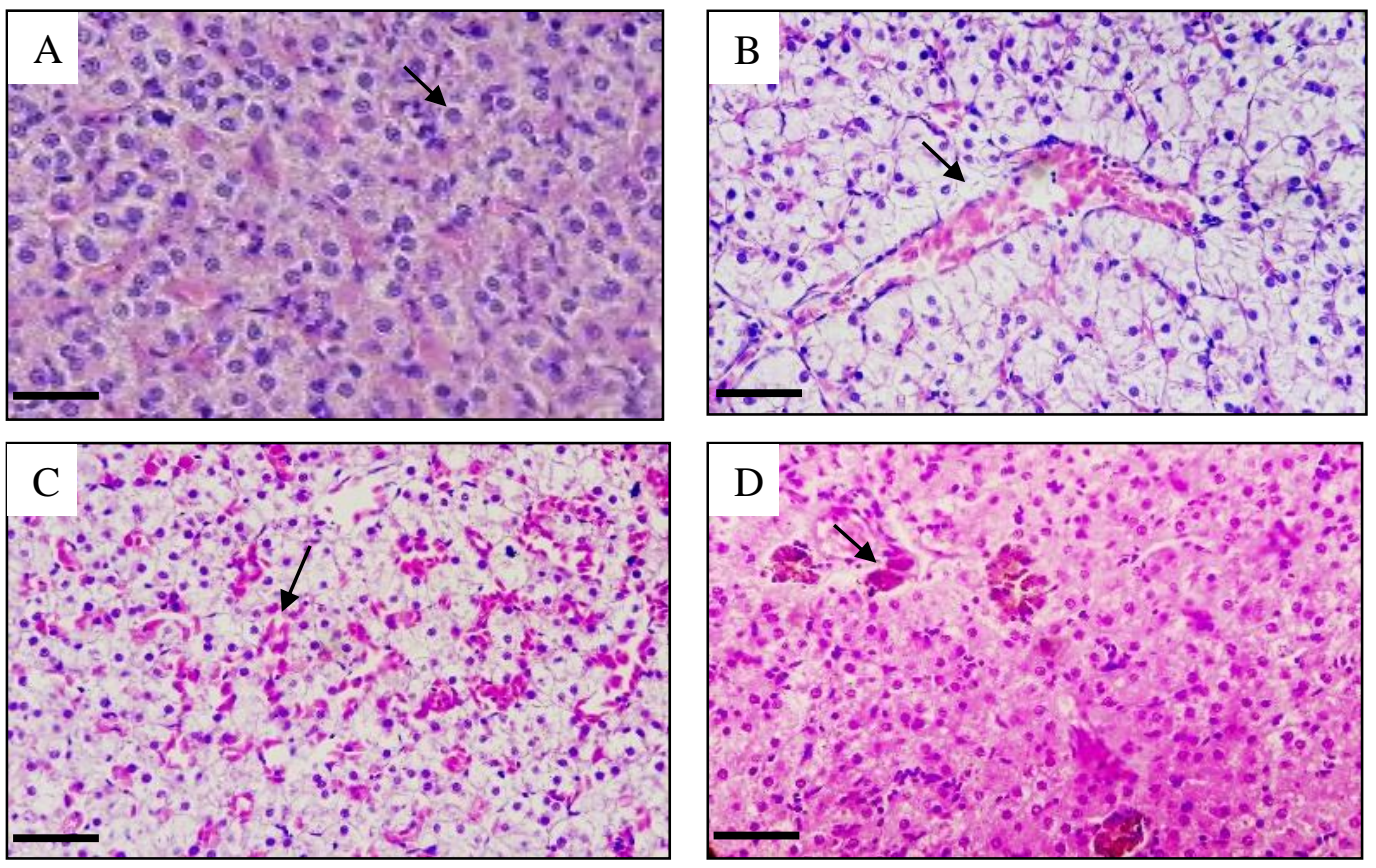

Figura 3. Fotomicrografias da região intermediária do fígado de S. herzbergii. A) Hepatócitos normais (seta); B) dilatação dos sinusoides (seta); C) congestão vascular (seta); D) melanamacrófagos (seta). Escala $=50 \mu \mathrm{m}$.

As brânquias dos peixes estão em contato direto com a água, desempenhando papéis importantes nos processos de respiração e manutenção do equilíbrio iônico e osmótico (Saleh e Marie, 2016). Esse contato constante com o meio circundante torna as brânquias o primeiro alvo de poluentes presentes no compartimento hídrico (Abdel-Moneim et al., 2012). Logo, as mudanças histológicas nesse órgão estão entre as principais respostas reconhecidas aos estressores ambientais. Por outro lado, o fígado desempenha importantes funções vitais, tais como a degradação metabólica de xenobióticos, o que, por sua vez, torna-o frequentemente suscetível a danos decorrentes de compostos tóxicos (Kostic et al., 2017). Assim, observou-se que tanto as brânquias como o fígado apresentaram diferentes respostas ao estresse ambiental. No entanto, as brânquias, por serem o primeiro órgão que entra em contato direto com a água, demonstraram ser o órgão mais afetado, com maior grau de alterações histológicas significativas.

Os dados morfométricos analisados demonstraram que os espécimes machos de $S$. herzbergii amostrados na Ilha dos Caranguejos apresentaram peso total superior aos capturados na região portuária $(\mathrm{P}=0,004)$. Esses dados indicam que existe maior incremento de massa corpórea nos indivíduos da área de referência, provavelmente, pelas condições mais adequadas de alimentação para a espécie na área (Tab. 2).

Tabela 2. Dados morfométricos (média \pm desvio-padrão) de Sciades herzbergii coletados em regiões estuarinas da Baía de São Marcos, Maranhão

\begin{tabular}{lllll}
\hline \multirow{2}{*}{ Parâmetros } & \multicolumn{3}{c}{ Média \pm Desvio-padrão } & A2 \\
\hline & Fêmeas $(\mathrm{n}=10)$ & Machos $(\mathrm{n}=10)$ & Fêmeas $(\mathrm{n}=6)$ & Machos $(14)$ \\
$\mathrm{CT}(\mathrm{cm})$ & $34,19 \pm 4,11$ & $31,05 \pm 6,57$ & $32,68 \pm 4,5$ & $27,49 \pm 5,71$ \\
CP $(\mathrm{cm})$ & $28,34 \pm 3,59$ & $26,06 \pm 3,11$ & $27,26 \pm 4,06$ & $23,8 \pm 2,88$ \\
CF $(\mathrm{cm})$ & $28,91 \pm 3,74$ & $26,33 \pm 2,99$ & $28,33 \pm 4,13$ & $25,37 \pm 2,69$ \\
PT $(\mathrm{g})$ & $318,66 \pm 96,97$ & $261,5 \pm 93,02 *$ & $275,33 \pm 108,7$ & $162,07 \pm 66,31 *$ \\
\hline
\end{tabular}

A1 (área referência); A2 (área potencialmente impactada); CT: comprimento total; CP: comprimento padrão; CF: comprimento furcal, PT: peso total; *diferenças significativas entre os locais de amostragem $(\mathrm{P}<0,05)$. 
Estudos realizados por Carvalho-Neta e AbreuSilva (2013), na Baía de São Marcos, evidenciaram que as concentrações de alumínio dissolvido, cádmio total, ferro dissolvido, manganês, chumbo, mercúrio total, benzeno, fenóis, tributilestanho e bifenilas policloradas, presentes nos sedimentos coletados próximo ao Porto do Itaqui, foram superiores aos limites aceitáveis pelos padrões nacionais (Brasil, 2005), confirmando, portanto, o caráter de contaminação química dessa região. Essa mesma situação de contaminação por metais pesados e compostos orgânicos foi registrada em outras pesquisas realizadas na região (Fortes Carvalho-Neta et al., 2016, 2019).

Nesse contexto, os contaminantes, quando inseridos no ambiente aquático, podem apresentar distribuições complexas, tais como coluna d'água, alimentos e sedimentos, sendo a exposição dos organismos aos agentes tóxicos dependente de como cada espécie se relaciona e coleta amostras de seu meio (Sardi et al., 2016). Como $S$. herzbergii é uma espécie de fundo e se alimenta de organismos bentônicos (Ribeiro et al., 2012), é provável que os peixes analisados estivessem submetidos à contaminação advinda destes três compartimentos: água, sedimento e alimentos.

A exposição dos organismos aquáticos aos contaminantes pode levar à redução ou à perda de vários sistemas biológicos, sejam bioquímico, fisiológico ou comportamental, além de demandar energia necessária para processos de detoxificação (Lam, 2009). Dessa maneira, outros parâmetros, como o aumento em peso, podem ficar comprometidos, e isso parece explicar os valores inferiores da média de peso total dos peixes machos coletados na região portuária aqui analisada.

É importante destacar que os machos da espécie $S$. herbergii incubam, na boca, os ovos fertilizados pelas fêmeas, e consequentemente sua alimentação é reduzida, visto que alocam quantidades significativas de energia para a reprodução (Queiroga et al., 2012). Assim, durante as coletas, foi observada a presença de ovos na cavidade bucal de alguns exemplares machos capturados na região potencialmente contaminada, que fica a uma certa distância $(10 \mathrm{~km})$ da principal movimentação industrial do porto público (Itaqui). Esses resultados foram diferentes dos encontrados por Fortes Carvalho Neta et al. (2016), que trabalharam com a mesma espécie em regiões mais próximas do Porto de Itaqui.

Segundo esses autores, o comportamento de incubar os ovos na boca não foi registrado nos peixes capturados próximo à área portuária, sendo este um indicativo de resposta comportamental dos organismos referente ao impacto antropogênico. Entretanto, é necessário enfatizar que determinados fatores ecológicos, que variam no tempo e no espaço, podem interferir nessa configuração. Um exemplo disso é a predação, seja natural ou antrópica, a competição por espaço e a variabilidade biológica dos indivíduos, que fazem com que alguns organismos de uma dada população habitem regiões mais impactadas, a fim de obter uma maior aptidão (Ricklefs e Relyea, 2016). No entanto, essa escolha pode resultar em custos biológicos, tal como a diminuição de peso corporal. Logo, a alimentação reduzida por conta da incubação dos ovos na boca associada às concentrações elevadas de poluentes podem ter influenciado no menor ganho de peso total dos peixes machos amostrados na área potencialmente impactada.

A avaliação macroscópica das gônadas revelou que $60 \%$ dos exemplares amostrados foram machos, e $40 \%$ fêmeas. Os machos capturados na Região Portuária demonstraram diferentes fases de desenvolvimento gonadal e esses achados diferiram dos registrados por Sousa et al. (2013), que não encontraram peixes imaturos para essa mesma região. Já as fêmeas de ambos os locais apresentaram a maioria das fases maturacionais (Tab. 3). No geral, houve predomínio de espécimes na fase de desenvolvimento (FII) para as duas regiões de coleta. Entretanto, não foram observados indivíduos na fase de regeneração.

Em relação às variáveis ambientais, verificou-se que os valores médios de temperatura, salinidade e pH não apresentaram diferença significativa $(\mathrm{P}<0,05)$ entre as áreas estudadas para os períodos chuvoso e de estiagem. Entretanto, houve diferença nos valores de $\mathrm{O}_{2}$ dissolvido entre os períodos sazonais, com $\mathrm{P}=0,04$ (Tab. 4). 
Tabela 3. Fases de desenvolvimento gonadal de S. herzbergii coletados em regiões da Baía de São Marcos, Maranhão

\begin{tabular}{|c|c|c|c|c|}
\hline \multirow[b]{2}{*}{ Fases } & \multicolumn{2}{|c|}{ A1 } & \multicolumn{2}{|c|}{ A2 } \\
\hline & Fêmeas & Machos & Fêmeas & Machos \\
\hline F I & $23 \%$ & $37 \%$ & $0 \%$ & $21 \%$ \\
\hline F II & $46 \%$ & $50 \%$ & $33 \%$ & $36 \%$ \\
\hline F III & $31 \%$ & $0 \%$ & $50 \%$ & $29 \%$ \\
\hline F IV & $0 \%$ & $13 \%$ & $17 \%$ & $14 \%$ \\
\hline F V & $0 \%$ & $0 \%$ & $0 \%$ & $0 \%$ \\
\hline
\end{tabular}

F I: imaturo; F II: desenvolvimento; F III: desova; F IV: regressão; FV: regeneração.

Tabela 4. Parâmetros abióticos registrados nos períodos chuvoso e de estiagem nas duas regiões estuarinas da Baía de São Marcos, Maranhão

\begin{tabular}{lccccl}
\hline & \multicolumn{2}{c}{ Chuvoso } & \multicolumn{3}{c}{ Estiagem } \\
Parâmetros & A1 & A2 & A1 & A2 & Valores recomendados \\
\hline Temperatura $\left({ }^{\circ} \mathrm{C}\right)$ & 29,5 & 28,8 & 29,1 & 28,7 & $28-32{ }^{\circ} \mathrm{C} \mathrm{a}$ \\
Salinidade & 13,3 & 15,3 & 16,5 & 24,6 & $\geq 30 \%$ a \\
pH & 7,46 & 7,52 & 7,37 & 7,78 & $6,5-8,5$ a \\
O2 dissolvido (mg/L) & $5,3^{*}$ & $4,6^{*}$ & $7,2^{*}$ & $6,7 *$ & $>4 \mathrm{mg} / \mathrm{L} \mathrm{a}$ \\
\hline
\end{tabular}

A1 (área referência); A2 (área potencialmente impactada); mg: miligramas; L: litros; ${ }^{*}=$ diferenças estatística entre os períodos sazonais $(\mathrm{P}<0,05)$; $\mathrm{a}=$ valores permitidos pela Resolução Conama n 357/2005 e nº 430/2011, água salobra, classe II.

Os dados abióticos encontrados neste estudo estão de acordo com os valores de referência indicados pela Resolução Conama para águas salobras (Brasil, 2011) e são considerados normais, conforme outros trabalhos realizados nessa região (Carvalho-Neta e Abreu-Silva, 2013; Sousa et al., 2013; Castro et al., 2018). O oxigênio dissolvido foi o único parâmetro analisado que apresentou diferenças significativas entre os períodos sazonais $(\mathrm{P}=0,04)$. Os maiores teores dessa variável ambiental ocorreram no período de estiagem, independentemente das áreas de coleta.

Esse elemento é indispensável à manutenção dos processos vitais dos animais e é o mais importante indicador da qualidade ambiental, visto que o grande aporte de contaminantes provenientes de efluentes industriais e domésticos causa um acentuado desequilíbrio dos parâmetros hidrológicos, principalmente aqueles relacionados com a diminuição do oxigênio dissolvido (Santos et al., 2009). Assim, os menores valores registrados dessa variável no período chuvoso podem indicar o aumento da carga de contaminantes que são facilmente carreados do continente para o ambiente aquático.

\section{CONCLUSÕES}

Os valores dos índices histológicos dos órgãos analisados demonstraram diferentes níveis de estresse dos peixes em pontos distintos da Baía de São Marcos, Maranhão. Os exemplares amostrados na região portuária apresentaram danos teciduais maiores, e as brânquias indicaram ser o órgão mais afetado. Apesar de inespecíficas, as alterações identificadas mostraram-se relevantes e ratificam as descobertas encontradas para a espécie estudada no estado do Maranhão, Brasil.

\section{AGRADECIMENTOS}

À Capes e à Fundação de Amparo à Pesquisa e Desenvolvimento Científico do Maranhão (Fapema), pelo auxílio financeiro; ao Laboratório de Biomarcadores em Organismos Aquáticos (LABOAq), pelo suporte físico para as análises dos organismos coletados.

\section{REFERÊNCIAS}

ABDEL-MONEIM, A.M.; AL-KAHTANI, M.A.; ELMENSHAWY, O.M. Histopathological biomarkers in gills and liver of Oreochromis niloticus from polluted wetland environments, Saudi Arabia. Chemosphere, v.88, p.1028-1035, 2012.

BERNET, D.; SCHMIDT, H.; MEIER, W. et al. Histopathology in fish: proposal for a protocol to assess. J. Fish Dis., v.22, p.25-34, 1999. 
BRASIL. Conselho Nacional do Meio Ambiente (CONAMA). Resolução n n $^{\circ}$ 357, de 17 de março de 2005. Dispõe sobre a classificação dos corpos de água e diretrizes ambientais para o seu enquadramento, bem como estabelece as condições e padrões de lançamento de efluentes, e dá outras providências. Diário Oficial da União, Brasília, 18 mar. 2005. p.58-63. Disponível em: <http://www.mma.gov.br/port/conama/res11/res 43011.pdf>. Acessado em: 20 fev. 2019.

BRASIL. Conselho Nacional do Meio Ambiente (CONAMA). Resolução $n^{\circ} 430$, de 13 de maio de 2011. Dispõe sobre as condições e padrões de lançamento de efluentes. Diário Oficial da União, Brasília. 13 maio 2011. Disponível em: <http://www.mma.gov.br/port/conama/res11/res 43011.pdf>. Acessado em: 20 fev. 2019.

BROWN-PETERSON, N.J.; WYANSKI, D.M.; SABORIDO-REY, F. et al. A Standardized terminology for describing reproductive development in fishes. Mar. Coast. Fish., v.3, p.52-70, 2011.

CAPUTO, L.F.G.; GITIRANA, L.B.; MANSO, P.P.A. Técnicas histológicas. In: MOLINARO, E.M.; CAPUTO, L.F.G.; AMENDOEIRA, M.R.R (Orgs.). Conceitos e métodos para a formação de profissionais em laboratórios de saúde. Rio de Janeiro: EPSJV, IOC, 2010. p.93185.

CARVALHO-NETA, R.N.F.; ABREU-SILVA, A.L. Glutathione S-Transferase as biomarker in Sciades herzbergii (Siluriformes: Ariidae) for environmental monitoring: the case study of São Marcos Bay, Maranhão Brasil. Lat. Am. J. Aquat. Res., v.41, p.217-225, 2013.

CARVALHO-NETA, R.N.F.; TORRES, A.R.JR.; ABREU-SILVA, A.L. Biomarkers in Catfish Sciades herzbergii (Teleostei: Ariidae) from polluted and non polluted areas (São Marcos' Bay, Northeastern Brazil). Appl. Biochem. Biotechnol., v.166, p.1-12, 2012.

CASTRO, J.S.; FRANÇA, C.L.; CARDOSO, R.L. et al. Histological changes in the kidney of Sciades herzbergii (Siluriformes, Ariidae) for environmental monitoring of a neotropical estuarine Area (São Marcos Bay, Northeastern Brazil). Bull. Environ. Contam. Toxicol., v.103, p.246-254, 2019.
CASTRO, J.S.; FRANÇA, C.L.; FERNANDES, J.F. et al. Biomarcadores histológicos em brânquias de Sciades herzbergii (Siluriformes, Ariidae) capturados no Complexo Estuarino de São Marcos, Maranhão. Arq. Bras. Med. Vet. Zootec., v.70, p.410-418, 2018.

CONSELHO Federal de Medicina Veterinária. Resolução 1000, de 11 de maio de 2012. Dispõe sobre procedimentos e métodos de eutanásia em animais, e dá outras providências. Disponível em: <http://portal.cfmv.gov.br/lei/index/id/326>. Acessado em: 25 jul. 2019.

FORTES CARVALHO NETA, R.N.F.; ANDRADE, T.S.O.M.; OLIVEIRA, S.R.R. et al. Biochemical and morphological responses in Ucides cordatus (Crustacea, Decapoda) as indicators of contamination status in mangroves and port areas from northern Brazil. Environ. Sci. Pollut. Res., v.26, p.15884-15893, 2019.

FORTES CARVALHO NETA, R.N.F.; BARBOSA, G.L.; TORRES, H.S. et al. Changes in glutathione S-Transferase activity and parental care patterns in a catfish (Pisces, Ariidae) as a biomarker of anthropogenic impact in a Brazilian Harbor. Arch. Environ. Contam. Toxicol., v.72, p.132-141, 2016.

KOSTIC. J.; KOLAREVIC. S.; KRACUNKOLAREVIC. M. et al. The impact of multiple stressors on the biomarkers response in gills and liver of freshwater breams during different seasons. Sci. Total Environ., v.601, p.1670-1681, 2017.

LAM, P.K.S. Use of biomarkers in environmental monitoring. Ocean Coastal Manag., v.52, p.348354, 2009.

PEREIRA FILHO, J.P.; SPILLERE, L.C.; SCHETTINI, C.A.F. Dinâmica de nutrientes na região portuária do estuário do Rio Itajaí-Açu, SC. Atlântica, v.25, p.11-20, 2003.

PINHEIRO-SOUSA, D.B. et al. A screening test based on hematological and histological biomarkers to evaluate the environmental impacts in tambaqui (Colossoma macropomum) from a protected area in Maranhão, Brazilian Amazon. Chemosphere, v.214, p.445-451, 2019.

PRABHAKARAN, K.; NAGARAJAN, R.; MERLIN FRANCO, F.; KUMAR, A.A. Biomonitoring of Malaysian aquatic environments: a review of status and prospects. Ecohydrol. Hydrobiol., v.17, p.134-147, 2017. 
QUALHATO, G.; SABÓIA-MORAIS, S.M.T.; SILVA, L.D.; ROCHA, L.T. Melanomacrophage response and hepatic histopathologic biomarkers in the guppy Poecilia reticulata exposed to iron oxide (maghemite) nanoparticles. Aquat. Toxicol., v.198, p.63-72, 2018.

QUEIROGA, F.R. et al. Reproductive biology of Sciades herzbergii (Siluriformes: Ariidae) in a tropical estuary in Brazil. Zoologia, v.29, p.397404, 2012.

RAINBOW, P.S. Biomonitoring of heavy metal availability in the marine environment. Mar. Pollut. Bull., v.31, p.183-192, 1995.

RAUTENBERG, G.E.; AMÉ, M.V.; MONFERRÁN, M.V. et al. A multi-level approach using Gambusia affinis as a bioindicator of environmental pollution in the middle-lower basin of Suquía River. Ecol. Indic., v.48, p.706720, 2015.

RIBEIRO, E.B.; ALMEIDA, Z.S.; CARVALHONETA, R.N.F. Hábito alimentar do bagre Sciades herzbergii (Siluriformes, Ariidae) da Ilha dos Caranguejos, Maranhão, Brasil. Arq. Bras. Med. Vet. Zootec., v.64, p.1761-1765, 2012.

RICKLEFS, R.; RELYEA, R.A. Economia da Natureza. 7.ed. Rio de Janeiro: Guanabara Koogan, 2016.
SALEH, Y.S.; MARIE, M.A.S. Use of Arius thalassinus fish in a pollution biomonitoring study, applying combined oxidative stress, hematology, biochemical and histopathological biomarkers: a baseline field study. Mar. Pollut. Bull., v.106, p.308-322, 2016.

SANTOS, T.G.; BEZERRA JUNIOR, J.L.; COSTA, K.M.P.; FEITOSA, F.A.N. Dinâmica da biomassa fitoplanctônica e variáveis ambientais em um estuário tropical (Bacia do Pina, Recife, PE). Rev. Bras. Eng. Pesca, v.4, p.95-109, 2009.

SARDI, A.E.; RENAUD, P.E.; LANA, P.C.; CAMUS, L. Baseline levels of oxidative stress biomarkers in species from a subtropical estuarine system (Paranaguá Bay, southern Brazil). Mar. Pollut. Bull., v.113, p.496-508, 2016.

SOUSA, D.B.P.; ALMEIDA, Z.S.; CARVALHO-NETA, R.N.F. Biomarcadores histológicos em duas espécies de bagre estuarinos da Costa Maranhense, Brasil. Arq. Bras. Med. Vet. Zootec., v.65, p.369-376, 2013.

STENTIFORD, G.D.; LONGSHAW, M.; LYONS, B.P. et al. Histopathological biomarkers in estuarine fish species for the assessment of biological effects of contaminants. Mar. Environ. Res., v.55, p.137-159, 2003. 